\title{
A GRASP-based approach to the multi activity combined timetabling and crew scheduling problem considering a heterogeneous workforce
}

\author{
Diego Novoa, Camilo Olarte, David Barrera* and Eliana María González-Neira
}

Department of Industrial Engineering, Pontificia Universidad Javeriana, Bogotá, Colombia, Cra. 7 No. 40-62 - Edificio José Gabriel Maldonado

\begin{tabular}{l}
\hline C H R O N I C L E \\
\hline Article history: \\
Received November 42015 \\
Received in Revised Format \\
April 62016 \\
Accepted April 72016 \\
Available online \\
April 72016 \\
\hline Keywords: \\
Workforce Scheduling \\
Multi-activity Combined \\
Timetabling and Crew Scheduling \\
Problem (MCTCSP) \\
Heterogeneous workforce \\
Categorical Skills \\
GRASP \\
\hline
\end{tabular}
A B S T R A C T

This paper tackles an extension to the Multi-activity Combined Timetabling and Crew Scheduling Problem (MCTCSP). The goal of the original problem is to schedule the minimum number of homogenous workers required, in order to visit a set of customers characterized by services needed against schedule availability. However, since in home services it is common to have specialized workers, a mathematical model considering a heterogeneous workforce is proposed. As a solution, a GRASP-based algorithm is designed. In order to test the metaheuristic performance, 110 instances from the literature are adapted to include categorical skills. In addition, another 10 instances are randomly generated to consider large problems. The results show that the proposed GRASP finds optimal solutions in $46 \%$ of the cases and saves $40-96 \%$ computational time.

\section{Introduction}

The Multi-activity Combined Time tabling and Crew Scheduling Problem (MCTCSP) was proposed by Barrera et al. (2012). Since it is an extension of the two NP-hard problems, MCTCSP can also be classified as NP-hard (Fischetti et al., 1989; Burke \& Petrovic, 2002; Azadeh et al., 2013). The MCTSP considers a set of customers, with different geographical locations, which requires a set of services. These services must be provided in the client's place of residence, by selecting one of their desirable schedules. To meet the requirements, a set of workers is available with identical skills. The purpose of this problem is to determine which one of the workers should visit each customer and at what time. To do so, a set of routes that consume the minimal amount of resources is built. Considering the framework proposed by Ernst et al. (2004), this can be classified as a line of work construction problem. According to Barrera et al. (2012) there are two innovative features in the problem: i) the need to decide when every client is served and ii) a multiple activities environment. This configuration was motivated by a case study in which medical staff visits schools. In this way, if a set of clients $\mathcal{C}$ is considered, each one will determine a subset of schedules $\mathrm{N}_{\mathrm{i}}$ in which it can be visited. To model this problem, a directed graph $\mathcal{G}\{\mathcal{N}, \mathcal{A}\}$ is

* Corresponding author. Tel: +57-1-3208320 Ext. 5307 Fax: +57-1-3208320 Ext. 5275

E-mail: barrera-o@javeriana.edu.co (D. Barrera)

(C) 2016 Growing Science Ltd. All rights reserved. doi: $10.5267 /$ j.ijiec. 2016.4 .001 
proposed. Within this graph, $\mathcal{N}$ is the set of nodes and $\mathcal{A}$ is the set of arcs. The set of nodes is formed by the union of the possible schedules of each costumer $\left(\mathcal{N}=\cup \mathrm{N}_{\mathrm{i}}, \forall \mathrm{i} \in \mathcal{C} \cup\{s\} \cup\{t\}\right)$ where $s$ and $t$ represent dummy nodes. Additionally, each node $\mathrm{j}(\mathrm{j} \in \mathcal{N})$ is associated with: a client $\mathrm{i}(\mathrm{i} \in \mathcal{C})$, a service $\mathrm{m}(\mathrm{m} \in \mathcal{S})$, a day $\mathrm{l}(\mathrm{l} \in \mathcal{D})$ and a start time for the visitn $(\mathrm{n} \in \mathcal{H})$. In the original problem, any worker $\mathrm{k}(\mathrm{k} \in \mathcal{K})$ is capable of providing any services $(\mathrm{s} \in \mathcal{S})$; that is, the workforce is considered homogeneous. In this context, the decision to be made is whether or not to use the $\operatorname{arc} \mathcal{A}(\mathrm{i}$. j). In spite of the fact that crew scheduling is a critical activity in service delivery environments (Sureshkumar \& Pillai, 2012, Hedjazi, 2015), the assumption of homogeneous workforce may involve difficulties for implementation (De Bruecker et al., 2015a). Consider, for example, the scenario in which some of the portfolio services are specialized. Consequently, there is a subset $S^{\prime}$ of services $\left(S^{\prime} \subset \mathcal{S}\right)$ that requires different skills. To use the modeling approach proposed in Barrera et al. (2012), the original optimization problem, that considers the set of services $\mathcal{S}$, must be divided in two sub problems. The first studies the set of services $S^{\prime}$; the second, the set $\overline{S^{\prime}}$ whenever $\mathcal{S}=S^{\prime} \cup \overline{S^{\prime}}$. In that case if $\widehat{f_{1}}=\min \left[\mathrm{f}_{1}(\mathrm{x}): \mathrm{x} \in \mathrm{S}\right]$, where $\widehat{\mathrm{f}_{1}}$ is the optimal value for the problem that includes all of the portfolio services and $\mathrm{x}$ represents the points of solution space, then it is expected that, for related portfolios, $\widehat{f_{1}} \leq \widehat{f_{2}}+\widehat{f_{3}}$, where $\widehat{f_{2}}$ and $\widehat{f_{3}}$ are the solutions for the two sub-problems. Consequently, when skills are incorporated, there is a greater potential for application of the solution (Parisio \& Jones, 2015). Fig. 1 represents an example of the graph $\mathcal{G}\{\mathcal{N}, \mathcal{A}\}$, which is based on the information from Table 1 . This example involves two clients, two services, five days, two schedule options for each customer and two types of workers. Client $A$ requests service 1 while Client $B$ needs both services. In addition, for Client $A$, service 1 can be provided on day 1 at 8:00 (node 1) or on day 3 at 10:00 (node 4). Finally, Worker 1 has the skills required to provide the two services, while Worker 2 can only provide service 2 .

Table 1

Data for example

\begin{tabular}{cccccc}
\hline Node & Customer & Service & Day & Hour & $\begin{array}{c}\text { Worker types that can provide the } \\
\text { service }\end{array}$ \\
\hline 1 & A & 1 & 1 & $8: 00$ & 1 \\
2 & B & 1 & 2 & $7: 00$ & 1,2 \\
3 & B & 2 & 3 & $7: 00$ & 1 \\
4 & A & 1 & 3 & $10: 00$ & 1 \\
5 & B & 1 & 4 & $11: 00$ & 1,2 \\
6 & B & 2 & 5 & $9: 00$ & 1,2 \\
\hline
\end{tabular}

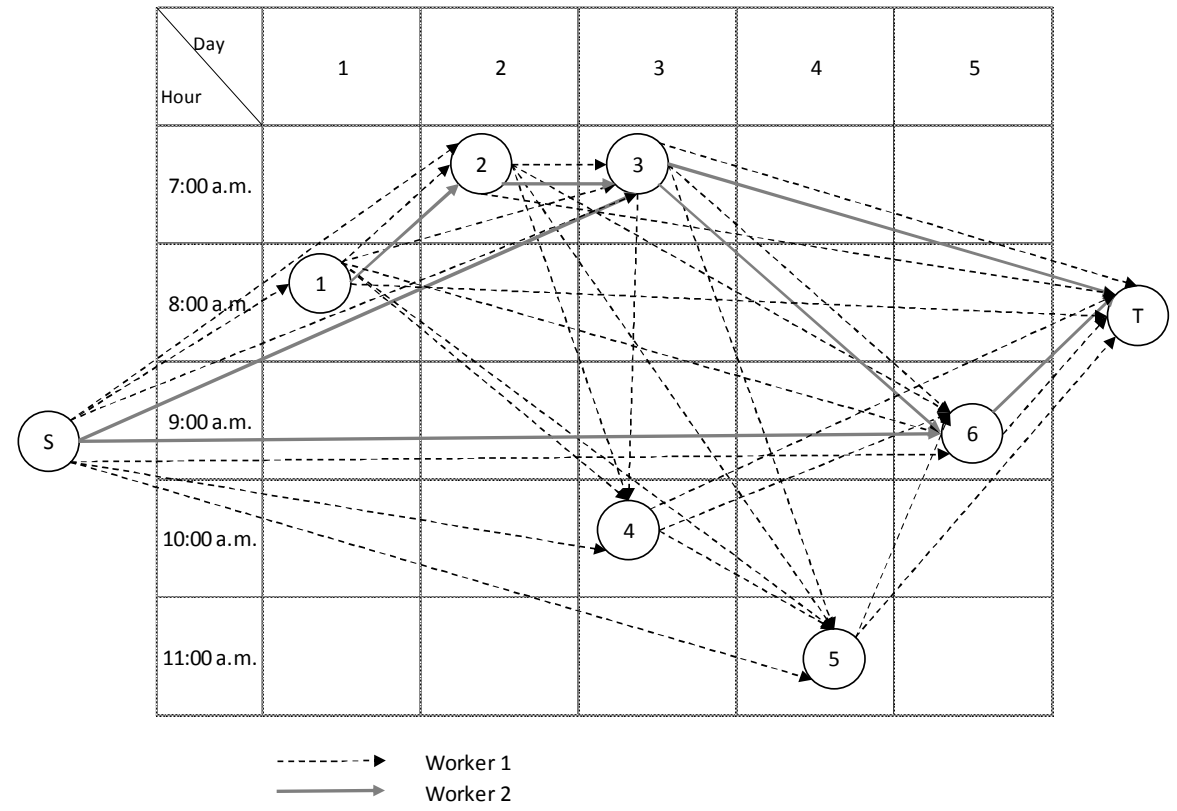

Fig. 1. Resulting graph for the example 
Three recent literature reviews conclude the relevance of such kinds of extensions in workforce scheduling problems (Van den Bergh et al., 2013; De Bruecker et al., 2015a; Pinedo et al., 2015). According to the authors it is necessary that, research in the area advances in two ways: i) include typical features of real systems to increase the impact, and ii) develop efficient algorithms as a way to close the gap between research and common practices in different industries. In this vein, multi-skilled workers have to be taken into consideration in order to find the optimal human resource allocation (Małachowski \& Korytkowski, 2016). According to Olivella \& Nembhard (2016), it is often most useful to obtain feasible solutions of the problem, even if they are suboptimal, rather than solving a relaxed version of the problem. Hence, recent applied research efforts, including skills modeling, cover a wide range of application areas (Henao et al., 2015).

As an example, Zacharia and Nearchou (2016), study the assembly line worker assignment and balancing problem (ALWABP). This problem seeks the best assignment of tasks to workers as well as the workers to workstations. Authors use categorical skills in order to model disabled workers planning. It is assumed that a worker found efficient to accomplish a particular set of tasks may be inefficient (or even unable to carry out) on another set. As a consequence, task times differ depending on worker skills. In this context, the proposed modeling approach allows to solve a real-world problem with particular social relevance.

On the other hand, Lieder et al. (2015) focus on home health care workforce planning. The authors include a preferred start time, for each task, which is surrounded by a penalty function for earliness and tardiness. Additionally, each task has a known duration and requires a certain qualification level (QL) of the respective care worker. Hence, the QLs are modeled as hierarchical skills. The results of this study show that, from a client perspective, using skill substitution is beneficial to the overall quality of service. Also, De Bruecker et al. (2015b) present a two-stage mixed integer programming approach for optimizing the skill mix and training schedule at an aircraft maintenance company. According to the authors, only workers that acquired the required license are allowed to maintain that aircraft. Hence, a categorical skills model is proposed.

Finally, Ladier (2014) propose a weekly timetabling and a daily rostering problem for warehouse personnel. Authors claim that, in logistics context, the qualifications are very specific to a person: two employees are very likely to have different skills and different licenses to drive the handling equipment. Therefore, the set of tasks mastered by a given employee will be different from the set of tasks mastered by any of his colleagues. In this context, clustering the employees according to their skills does not simplify the problem. Hence, skills modeling in classic rostering problems, it is an interesting research field for the next years in different industries (Chanpanit \& Udomsakdigool, 2015; Parisio \& Jones, 2015; Braekers et al., 2016).

This paper proposes an extension to MCTCSP so that workers with different skills are considered (MCTCSP-HW). In other contexts, similar extensions have been documented as site-dependent versions of classical problems (Zare-Reisabadi \& Hamid Mirmohammadi, 2015). According to the classification proposed in De Bruecker et al. (2015a), the MCTCSP-HW studies categorical skills. This means that one service only can be provided by workers with a particular skill. In problems where the decision concerns the number of workers, this extension has two implications: a greater number of decision variables and new coverage constraints. Finally, the construction of lines of work is operational (Gutiérrez et al., 2014) and in some environments may be dynamic. Hence, the NP-hard nature of the MCTCSP-HW supports the use of a meta-heuristic solution approach. Moreover, since in the MCTCSP-HW it is possible having large infeasible regions in the search space, GRASP is an appropriate method (Glorieux et al., 2015). On the other hand, constructive procedures combined with local search are a very effective solution method for some timetabling problems (Merlot et al., 2003). Thus, GRASP allows to produce feasible solutions for large instances more efficiently 
The remainder of the paper is organized as follows: Section 2 shows the mathematical model for the extended problem; Section 3 describes the solution strategy; and Sections 4 and 5 present computational experiments and concluding remarks, respectively.

\section{Integer programming model for MCTCSP-HW}

The extension of the model proposed in Barrera et al. (2012) considers the same definition for the graph $\mathcal{G}\{\mathcal{N}, \mathcal{A}\}$, detailed in Section 1. This is a directed and pre-processed graph so that the $\operatorname{arc} \mathcal{A}$ (i. j) exists only if the start time at node $j$ is later than the finishing time at node $i$, plus the traveling time between both nodes. The sets of nodes $(\mathcal{N})$, arcs $(\mathcal{A})$, clients $(\mathcal{C})$ and services $(\mathcal{S})$ are defined. Finally, in order to model the skills, a set of types of worker $(\mathcal{K})$ is included.

Additionally, it is known that a client $c$ demands a service $m$, which is represented by the binary parameter $b_{c m}$. The skills of a worker are included by the binary parameter $w_{i j k}$ that takes the value 1 if the worker type $k$ can use the $\operatorname{arc} \mathcal{A}(\mathrm{i}$. j); that is, if he/she has the required skills for providing the services at nodes $i$ and $j$. Finally, the nodes' information is modeled by the parameter $a_{j c m}$ that takes the value of 1 if the node $j$ corresponds to client $c$ and service $m$. The decision variable is $x_{i j k}$, which takes the value of 1 if a worker type $k$ uses the $\operatorname{arc} \mathcal{A}(\mathrm{i} . \mathrm{j})$.

$$
\min z: \quad \sum_{k \in \mathcal{K}} \sum_{j \in \mathcal{N}} x_{1 j k}
$$

subject to:

$$
\begin{aligned}
& \sum_{i \in \mathcal{N} \mid \mathcal{A}(\mathrm{i} . \mathrm{j}) \in \mathcal{A}} x_{i j k}-\sum_{i \in \mathcal{N} \mid \mathcal{A}(\mathrm{i} . \mathrm{j}) \in \mathcal{A}} x_{j i k}=1 \quad \forall j \in \mathcal{N} \\
& \sum_{i \in \mathcal{N}} \sum_{j \in \mathcal{N}} \sum_{\mathcal{A}(\mathrm{i} . \mathrm{j}) \in \mathcal{A}} x_{k \in \mathcal{K}} * a_{i c m}=b_{c m} \quad \forall c \in \mathcal{C}, \forall m \in \mathcal{S} \\
& x_{i j k} \leq w_{i j k} \quad \forall i \in \mathcal{N}, \forall j \in \mathcal{N} \mid \mathcal{A}(\mathrm{i} . \mathrm{j}) \in \mathcal{A}, \forall k \in \mathcal{K} \\
& x_{i j k} \in\{0,1\} \quad \forall i \in \mathcal{N}, \forall j \in \mathcal{N} \mid \mathcal{A}(\mathrm{i} . \mathrm{j}) \in \mathcal{A}, \forall k \in \mathcal{K}
\end{aligned}
$$

The objective function given in Eq. (1) is the minimization of the number of workers calculated as the number of arcs leaving the source node. The set of constraints represented in Eq. (2) guarantees that worker balance exists at each node. Eq. (3) guarantees the satisfaction of demand. Finally, Eq. (4) guarantees that workers without the required skills for providing a service are not assigned. The last set of constraints (Eq. (5)) defines the nature of the decision variables.

\section{A GRASP-based approach}

GRASP is a multi-start and iterative metaheuristic for combinatorial problems, in which each iteration consists of two phases: construction and local search (Resende \& Ribeiro, 2010). Fig. 2 describes the proposed algorithm for the MCTCSP-HW. In the construction phase, the algorithm starts by selecting one type of worker who can provide a service that no other can. In the case that this does not exist, the type of worker that provides more services of those requested is selected. After selecting the type of worker, visits are randomly assigned within a specified size of RCL. The RCL is constructed taking into account the relations of precedence. Since the graph is directed and oriented, when it is not possible to assign more visits, a new worker is selected.

The local search phase aims to reduce the number of workers. Initially it selects the worker with the least amount of assigned services, and attempts to allocate their visits to other workers of the solution. If that is not possible, it selects the next least used. This procedure continues until one of two stopping criteria is met: i) all the workers have been examined or ii) the process has reached a maximum runtime. 
In order to set the parameters of GRASP, an Experimental Design was carried out. According with Ridge \& Kudenko (2010) Design of Experiments (DOE) is an efficient way for tuning parameters in terms of the amount of data that need to be collected. This fact is critical to comprehend the algorithm design spaces. Authors analyze a case study and demonstrate the benefits of using DOE for parametrizing an Ant Colony to solve a Travelling Salesman Problem. Having this in mind, a nested three-factor experiment was designed.

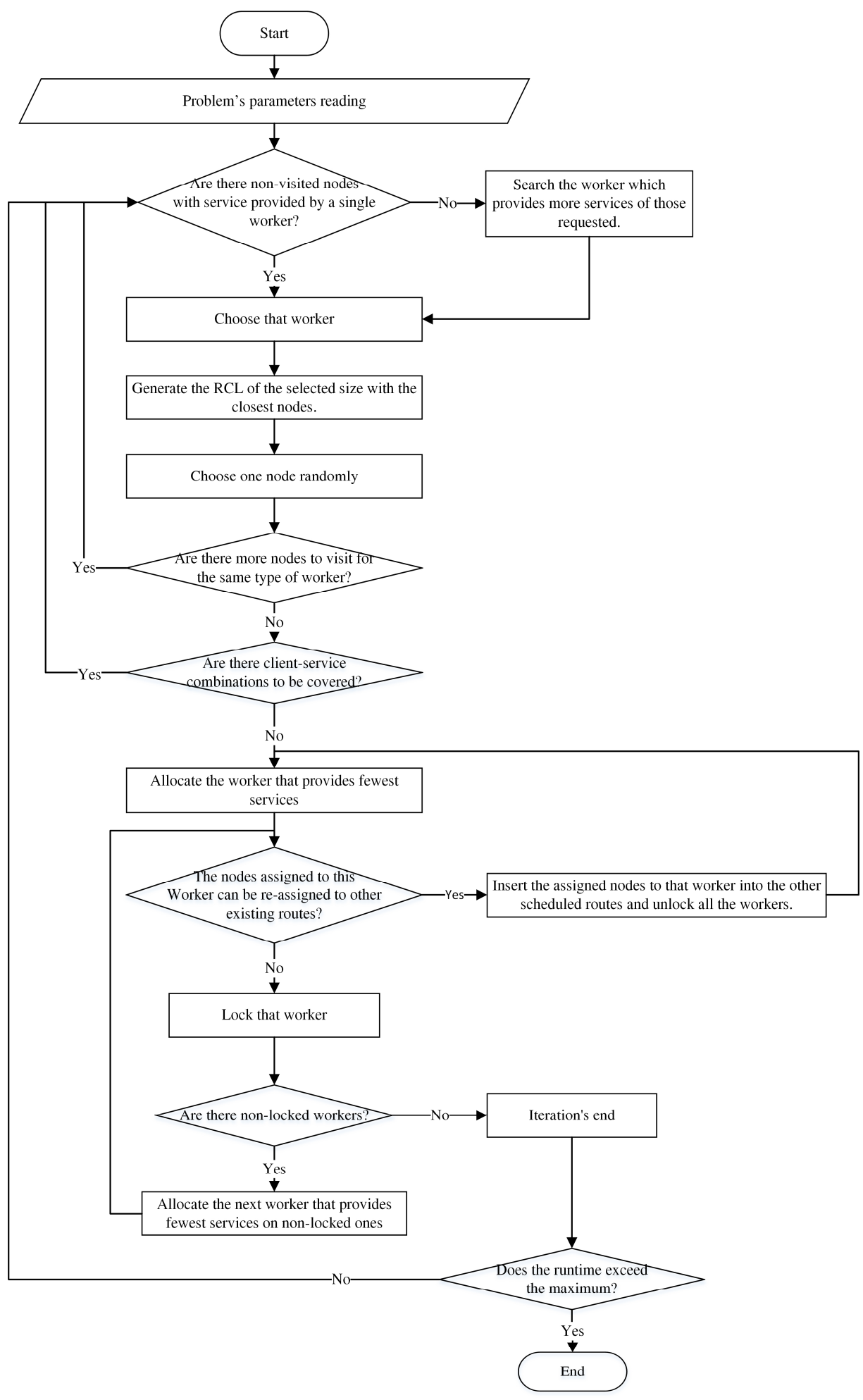

Fig. 2. Algorithm for the MCTCSP-HW 
The factor $A$ is the instance's size, factor $B$ the runtime and factor $C$ the RCL's size. The levels of factor $B$ are nested to factor $A$. From the runtime of the linear programming model, three levels of factor $A$ were defined: 150,300 and 900 nodes. As factor $B$ is nested in $A$, their levels are distinct for each instance's size. For instances of 150 nodes, it has the levels 5, 15 and $25 \mathrm{~s}$; for instances of 300 nodes, 5, 25 and $45 \mathrm{~s}$; and for instances of 900 nodes, 5,70 and $135 \mathrm{~s}$. As a final point, factor $C$ has the levels 3,6 and 9. Ten observations were analyzed for each treatment. The ANOVA allows us to conclude that the three factors A, B and C significantly influence the results of the GRASP (values-p of 3.2E-37, 3.6E-13 and 1.9E-2 respectively). To check the assumptions of the ANOVA, the normality (Shapiro-Wilks) and homogeneity of variances (Bartlett) tests were performed. Since the experiment did not meet the assumptions, non-parametric tests of Mood's means difference were performed.

For instances of 150 nodes, there is no significant difference between the resultant workers under 15 and $25 \mathrm{~s}$ of execution ( $\mathrm{p}$-value $=0.448$ ). Nonetheless, with a $\mathrm{p}$-value $<0.001$ the number of workers is less significant than the results obtained with a runtime of $5 \mathrm{~s}$. Thus, the selected runtime was $15 \mathrm{~s}$. A similar analysis was carried out for instances of 300 and 900 nodes. The conclusion is that the stop time for the metaheuristic was 15, 25 and 70 s respectively. Interaction plots are shown in Fig. 3.
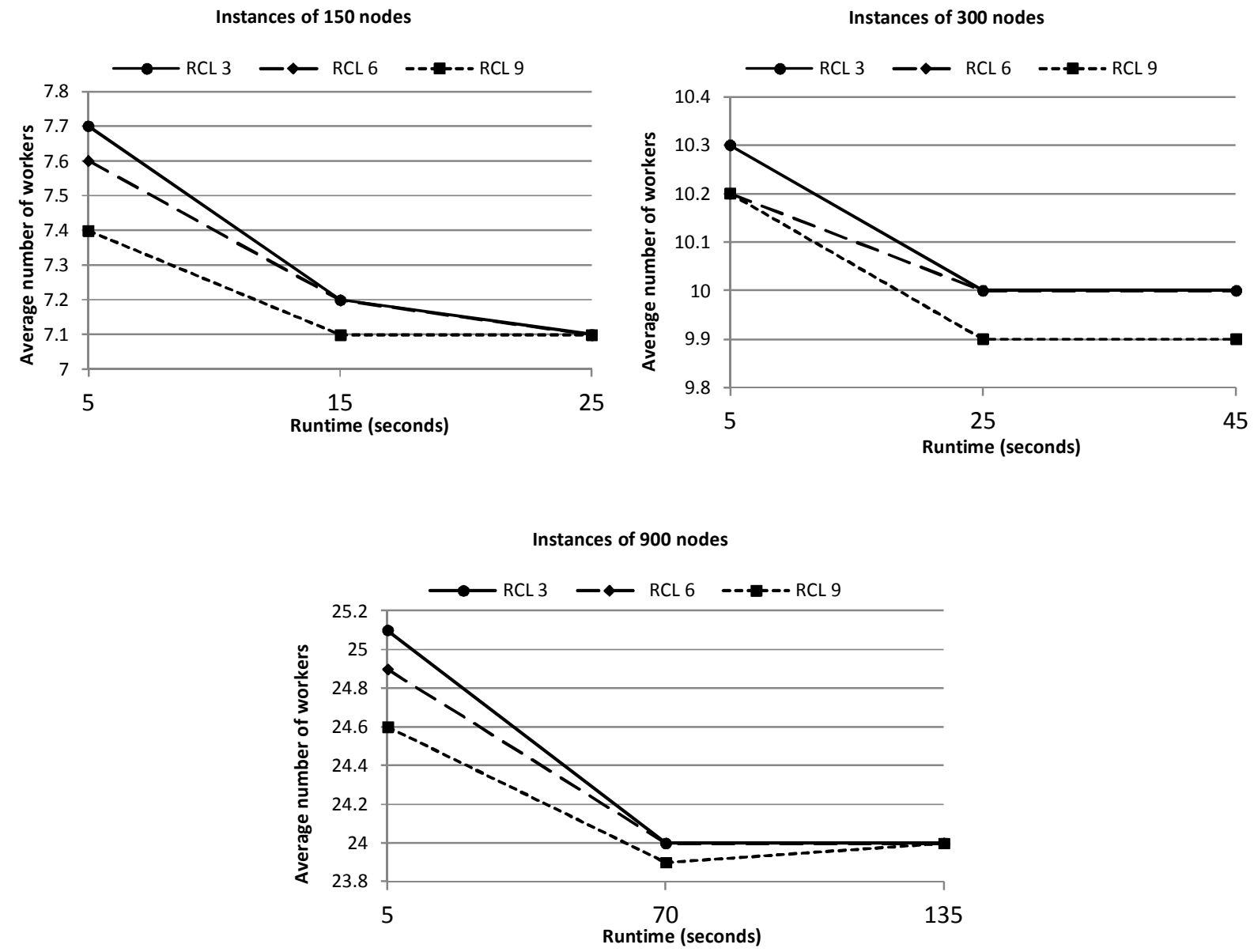

Fig. 3. Interaction plots Runtime (B) - RCL size (C)

\section{Computational Experiments}

For the experiment, instances reported in Barrera et al. (2012) were used. Those instances were randomly generated using information from a real problem. Additionally, in order to prove the extension at larger problems, 10 instances of 300 client-service combinations ( 900 nodes) were generated. Due to the nature 
of MCTCSP-HW, it was necessary to generate an additional parameter that associates a set of five types of worker with certain services. The generation of this parameter was random, ensuring two things: i) all requested services must be provided by at least one type of worker, and ii) the skills portfolio will cross at least two types of worker.

The model was implemented on Gusek and run on a computer Intel Xeon E5-2667 of $2.9 \mathrm{GHz}$ and 256 GB RAM.

Table 2 reports the average objective function, for each size of the problem, in the instances that were solved to optimality within $3600 \mathrm{~s}$. Thus, for example, of the instances of 35 client-service combinations (105 nodes), it was possible to solve eight within the proposed runtime of Barrera et al. (2012). The average time of those instances was $28.6 \mathrm{~s}$ and the objective function was 5.4 workers.

Table 2

Instances solved to optimality

\begin{tabular}{cccc}
\hline Instance's size & Average runtime(s) & Objective Function & Number of instances \\
\hline 35 & 28.6 & 5.4 & 8 \\
40 & 109.6 & 6.3 & 8 \\
45 & 107.6 & 6.6 & 10 \\
50 & 64.4 & 7.1 & 10 \\
55 & 53.5 & 7.5 & 6 \\
60 & 91.0 & 7.6 & 9 \\
65 & 100.0 & 7.9 & 8 \\
70 & 192.8 & 8.4 & 9 \\
75 & 185.7 & 8.9 & 7 \\
80 & 706.6 & 9.6 & 7 \\
100 & 453.4 & 8.3 & 8 \\
\hline
\end{tabular}

For some instances it was not possible to find an optimal solution within $3600 \mathrm{~s}$. Table 3 reports the best integer solution found by the model and the GAP of the solution regarding the relaxed problem. This means that, for example, for instances of 75 client-service combinations, there were three that found integer solutions within $3600 \mathrm{~s}$. Those solutions have an average objective function of 9.7 workers and a GAP of $20 \%$.

Table 3

Best integer solution and GAP within $3600 \mathrm{~s}$

\begin{tabular}{cccc}
\hline Instance's size & Objective Function & GAP & Number of instances \\
\hline 35 & 6.0 & $39 \%$ & 1 \\
40 & 8.0 & $59 \%$ & 2 \\
55 & 8.0 & $24 \%$ & 3 \\
60 & 8.0 & $24 \%$ & 1 \\
65 & 9.0 & $19 \%$ & 2 \\
70 & 9.0 & $20 \%$ & 1 \\
75 & 9.7 & $20 \%$ & 3 \\
80 & 10.0 & $15 \%$ & 1 \\
\hline
\end{tabular}

Finally, for instances of 900 nodes, it was not possible to find any integer solution within $3600 \mathrm{~s}$. Table 4 reports the results of the relaxed model. These results are especially interesting, because one of the main challenges is the design of algorithms that allow a quality solution in reasonable computational time (De Bruecker et al., 2015a). In large instances, as was expected, obtaining exact solutions is not an option. However, the purpose of this experiment is to establish comparison values that allow conclusions to be drawn about the quality of the solutions found with GRASP.

\section{Table 4}

Results of relaxed model with large instances

\begin{tabular}{lcccccccc}
\hline Instance & $300-1$ & $300-2$ & $300-3$ & $300-4$ & $300-5$ & $300-6$ & $300-7$ & $300-8$ \\
\hline Objective Function & 17.1 & 20.6 & 20.3 & 20.4 & 19.7 & 19.0 & 18.5 & 21.6 \\
\hline
\end{tabular}


Table 5 summarizes the results of GRASP and found optimal solutions. As shown, in instances of 35 customer-service combinations, using GRASP means a saving of $47.5 \%$ of the computational effort compared to $4.1 \%$ of sacrifice in the objective function. For the first five instances' sizes it was possible to find solutions with GAP below 5\%. Finally, in instances of 40 and 45 customer-service combinations GRASP found optimal solutions in $100 \%$ of cases. Similar results were found with the instances that could not be solved within $3600 \mathrm{~s}$ or less. For these cases, the saving of computational time is up to $96 \%$.

Table 5

Comparison with instances solved within $3600 \mathrm{~s}$

\begin{tabular}{|c|c|c|c|c|c|c|c|}
\hline \multirow{2}{*}{$\begin{array}{l}\text { Instance's } \\
\text { size }\end{array}$} & \multicolumn{2}{|c|}{ Exact solution } & \multicolumn{3}{|c|}{ GRASP } & \multirow{2}{*}{$\begin{array}{l}\text { Computational } \\
\text { Saving }\end{array}$} & \multirow{2}{*}{ Optimal \% } \\
\hline & Time $(\mathrm{s})$ & O.F & Time $(\mathrm{s})$ & O.F & GAP & & \\
\hline 35 & 28.56 & 5.44 & 15 & 5.67 & $4.1 \%$ & $47.5 \%$ & $78 \%$ \\
\hline 40 & 109.63 & 6.25 & 15 & 6.25 & $0.0 \%$ & $86.3 \%$ & $100 \%$ \\
\hline 45 & 107.60 & 6.60 & 15 & 6.60 & $0.0 \%$ & $86.1 \%$ & $100 \%$ \\
\hline 50 & 64.44 & 7.11 & 15 & 7.33 & $3.1 \%$ & $76.7 \%$ & $80 \%$ \\
\hline 55 & 53.50 & 7.50 & 25 & 7.67 & $2.2 \%$ & $53.3 \%$ & $83 \%$ \\
\hline 60 & 91.00 & 7.56 & 25 & 8.33 & $10.3 \%$ & $72.5 \%$ & $22 \%$ \\
\hline 65 & 100.00 & 7.86 & 25 & 8.71 & $10.9 \%$ & $75.0 \%$ & $13 \%$ \\
\hline 70 & 192.78 & 8.44 & 25 & 9.44 & $11.8 \%$ & $87.0 \%$ & - \\
\hline 75 & 185.71 & 8.86 & 25 & 10.00 & $12.9 \%$ & $86.5 \%$ & - \\
\hline 80 & 706.57 & 9.57 & 25 & 10.71 & $11.9 \%$ & $96.5 \%$ & $14 \%$ \\
\hline 100 & 453.38 & 8.30 & 25 & 9.75 & $17.5 \%$ & $94.5 \%$ & - \\
\hline
\end{tabular}

Another interesting comparison is the performance of the metaheuristic in instances where it was not possible to find optimal solutions. Table 6 presents the results compared among GRASP and the best solutions found in the entire maximum runtime. As shown, only in two instances is there a difference between the two solutions. Finally, in instances where integer solutions could not be found, GRASP reports an average GAP of $23 \%$ regarding the relaxed problem. Although the data are not directly comparable, it is important to note that for the second group of instances, the best solution for the linear programming model has an average GAP of $28 \%$.

Table 6

Comparison among GRASP and best integer solutions

\begin{tabular}{ccccccc}
\hline \multirow{2}{*}{ Instance } & \multicolumn{2}{c}{ Exact Solution } & \multicolumn{2}{c}{ GRASP } & \multicolumn{2}{c}{ Comparison } \\
& O.F & GAP & Time (s) & O.F. & Time (s) & O.F \\
\hline $35-7$ & 6 & $39.2 \%$ & 15 & 6 & 3585 & 0 \\
$40-2$ & 8 & $69.9 \%$ & 15 & 7 & 3585 & 1 \\
$40-5$ & 8 & $47.9 \%$ & 15 & 7 & 3585 & 1 \\
$55-5$ & 8 & $17.6 \%$ & 25 & 8 & 3575 & 0 \\
$55-7$ & 8 & $31.5 \%$ & 25 & 8 & 3575 & 0 \\
$55-8$ & 8 & $22.3 \%$ & 25 & 8 & 3575 & 0 \\
$60-8$ & 8 & $23.6 \%$ & 25 & 8 & 3575 & 0 \\
$65-3$ & 9 & $23.3 \%$ & 25 & 9 & 3575 & 0 \\
$65-6$ & 9 & $15.4 \%$ & 25 & 9 & 3575 & 0 \\
$70-4$ & 9 & $20.0 \%$ & 25 & 9 & 3575 & 0 \\
$75-1$ & 9 & $13.9 \%$ & 25 & 9 & 3575 & 0 \\
$75-6$ & 10 & $24.2 \%$ & 25 & 10 & 3575 & 0 \\
$75-8$ & 10 & $21.5 \%$ & 25 & 10 & 3575 & 0 \\
$80-5$ & 10 & $15.1 \%$ & 25 & 10 & 3575 & 0 \\
\hline
\end{tabular}

\section{Conclusions}

This paper proposed an extension to a problem in which workforce scheduling for home services is considered. The original version of the problem deals with homogeneous workers. However, in the context of service delivery, it is common to find that workers are specialized. Consequently, the original modeling strategy may involve sacrifices in the objective function in environments with related 
portfolios. Hence, a formulation that includes workers with categorical skills is proposed. Additionally a GRASP-based solution technique, for larger instances, is designed. This extension responded to what recent reviews in the field have considered potentially interesting future lines of work.

The proposed mathematical formulation is able to solve $75 \%$ of instances to optimality within less time than the maximum proposed by Barrera et al. (2012). The remaining $25 \%$ is divided into two groups: first, for $16 \%$ of the instances integer solutions were found. The average GAP of these solutions, regarding the relaxed problem, is $28 \%$. On the other hand, for $9 \%$ of the instances it was not possible to integer find solutions within the limited runtime. In this case, the problem was solved by relaxing the constraint of variables' integrality.

For instances where it was not possible to find optimal solutions in good computational time, a GRASPbased algorithm is proposed as a solution technique. From 120 instances tested, GRASP found optimal solutions in $46 \%$. Additionally, in $46 \%$ of instances, the difference between the exact solution and the one found by the metaheuristic is one worker; that is, the minimum difference. In terms of computational time, using GRASP, savings vary between $40-96 \%$.

This approach is particularly relevant for real-life applications. According to Barrera et al. (2012), stochastic times must be considered. It is therefore necessary to design efficient solution strategies that can be articulated with Monte Carlo Simulation (Juan et al., 2015). The designed GRASP-based algorithm saves up to $96.5 \%$ of computational effort.

\section{References}

Azadeh, A., Farahani, M. H., Eivazy, H., Nazari-Shirkouhi, S., \& Asadipour, G. (2013). A hybrid metaheuristic algorithm for optimization of crew scheduling. Applied Soft Computing, 13(1), 158-164.

Barrera, D., Velasco, N., \& Amaya, C. A. (2012). A network-based approach to the multi-activity combined timetabling and crew scheduling problem: Workforce scheduling for public health policy implementation. Computers \& Industrial Engineering, 63(4), 802-812.

Braekers, K., Hartl, R. F., Parragh, S. N., \& Tricoire, F. (2016). A bi-objective home care scheduling problem: Analyzing the trade-off between costs and client inconvenience. European Journal of Operational Research, 248(2), 428-443.

Burke, E. K., \& Petrovic, S. (2002). Recent research directions in automated timetabling. European Journal of Operational Research, 140(2), 266-280.

Chanpanit, T., \& Udomsakdigool, A. (2015). Workforce Planning for Single Call Center with ServiceLevel Agreement. In Toward Sustainable Operations of Supply Chain and Logistics Systems (pp. 521533). Springer International Publishing.

De Bruecker, P., Van den Bergh, J., Beliën, J., \& Demeulemeester, E. (2015a). Workforce planning incorporating skills: state of the art. European Journal of Operational Research, 243(1), 1-16.

De Bruecker, P., Van den Bergh, J., Belien, J., \& Demeulemeester, E. (2015b). A two-stage mixed integer programming approach for optimizing the skill mix and training schedules for aircraft maintenance. Available at SSRN 2697491.

Ernst, A. T., Jiang, H., Krishnamoorthy, M., \& Sier, D. (2004). Staff scheduling and rostering: A review of applications, methods and models. European journal of operational research, 153(1), 3-27.

Fischetti, M., Martello, S., \& Toth, P. (1989). The fixed job schedule problem with working-time constraints. Operations Research, 37(3), 395-403.

Glorieux, E., Danielsson, F., Svensson, B., \& Lennartson, B. (2015). Constructive cooperative coevolutionary optimisation for interacting production stations. The International Journal of Advanced Manufacturing Technology, 80(1-4), 673-688.

Gutiérrez, E. V., Galvis, O. D., López, D. A., Mock-Kow, J. S., Zapata, I., \& Vidal, C. J. (2014). Gestión logística en la prestación de servicios de hospitalización domiciliaria en el Valle del Cauca: caracterización y diagnóstico. Estudios Gerenciales, 30(133), 441-450.

Hedjazi, D. (2015). Scheduling a maintenance activity under skills constraints to minimize total weighted 
tardiness and late tasks. International Journal of Industrial Engineering Computations, 6(2), 135.

Henao, C. A., Muñoz, J. C., \& Ferrer, J. C. (2015). The impact of multi-skilling on personnel scheduling in the service sector: a retail industry case. Journal of the Operational Research Society, 66(12), 19491959.

Juan, A. A., Faulin, J., Grasman, S. E., Rabe, M., \& Figueira, G. (2015). A review of simheuristics: Extending metaheuristics to deal with stochastic combinatorial optimization problems. Operations Research Perspectives, 2, 62-72.

Ladier, A. L. (2014). Scheduling cross-docking operations: Integration of operational uncertainties and resource capacities (Doctoral dissertation, Université de Grenoble).

Lieder, A., Moeke, D., Koole, G., \& Stolletz, R. (2015). Task scheduling in long-term care facilities: A client-centered approach. Operations Research for Health Care, 6, 11-17.

Małachowski, B., \& Korytkowski, P. (2016). Competence-based performance model of multi-skilled workers. Computers \& Industrial Engineering, 91, 165-177.

Merlot, L. T., Boland, N., Hughes, B. D., \& Stuckey, P. J. (2002). A hybrid algorithm for the examination timetabling problem. In Practice and theory of automated timetabling IV (pp. 207-231). Springer Berlin Heidelberg.

Olivella, J., \& Nembhard, D. (2016). Calibrating cross-training to meet demand mix variation and employee absence. European Journal of Operational Research, 248(2), 462-472.

Parisio, A., \& Jones, C. N. (2015). A two-stage stochastic programming approach to employee scheduling in retail outlets with uncertain demand. Omega, 53, 97-103.

Pinedo, M., Zacharias, C., \& Zhu, N. (2015). Scheduling in the service industries: An overview. Journal of Systems Science and Systems Engineering, 24(1), 1-48.

Resende, M. G., \& Ribeiro, C. C. (2010). Greedy randomized adaptive search procedures: Advances, hybridizations, and applications. In Handbook of metaheuristics (pp. 283-319). Springer US.

Ridge, E., \& Kudenko, D. (2010). Tuning an algorithm using design of experiments. In Experimental methods for the analysis of optimization algorithms (pp. 265-286). Springer Berlin Heidelberg.

Sureshkumar, M., \& Pillai, V. (2012). Planning annuaulised hours when spike in demand exists. International Journal of Industrial Engineering Computations, 3(3), 313-320.

Van den Bergh, J., Beliën, J., De Bruecker, P., Demeulemeester, E., \& De Boeck, L. (2013). Personnel scheduling: A literature review. European Journal of Operational Research, 226(3), 367-385.

Zacharia, P. T., \& Nearchou, A. C. (2016). A population-based algorithm for the bi-objective assembly line worker assignment and balancing problem. Engineering Applications of Artificial Intelligence, 49, 1-9.

Zare-Reisabadi, E., \& Mirmohammadi, S. H. (2015). Site dependent vehicle routing problem with soft time window: Modeling and solution approach. Computers \& Industrial Engineering, 90, 177-185. 